\title{
Comparison of visual and automated Deki Reader interpretation of malaria rapid diagnostic tests in rural Tanzanian military health facilities
}

Akili K. Kalinga ${ }^{1,8^{*}}$, Charles Mwanziva ${ }^{3}$, Sarah Chiduo ${ }^{2}$, Christopher Mswanya ${ }^{3}$, Deus I. Ishengoma ${ }^{4}$, Filbert Francis ${ }^{4}$, Lucky Temu², Lucas Mahikwano², Saidi Mgata², George Amoo', Lalaine Anova ${ }^{5}$, Eyako Wurrapa ${ }^{5}$, Nora Zwingerman 6 , Santiago Ferro ${ }^{6}$, Geeta Bhat ${ }^{6}$, lan Fine ${ }^{6}$, Brian Vesely ${ }^{5}$, Norman Waters ${ }^{5}$, Mara Kreishman-Deitrick ${ }^{5}$, Mark Hickman ${ }^{5}$, Robert Paris ${ }^{5}$, Edwin Kamau ${ }^{5}$, Colin Ohrt ${ }^{5,9}$ and Reginald A. Kavishe

\begin{abstract}
Background: Although microscopy is a standard diagnostic tool for malaria and the gold standard, it is infrequently used because of unavailability of laboratory facilities and the absence of skilled readers in poor resource settings. Malaria rapid diagnostic tests (RDT) are currently used instead of or as an adjunct to microscopy. However, at very low parasitaemia (usually $<100$ asexual parasites/ $\mu$ l), the test line on malaria rapid diagnostic tests can be faint and consequently hard to visualize and this may potentially affect the interpretation of the test results. Fio Corporation (Canada), developed an automated RDT reader named Deki Reader ${ }^{\mathrm{TM}}$ for automatic analysis and interpretation of rapid diagnostic tests. This study aimed to compare visual assessment and automated Deki Reader evaluations to interpret malaria rapid diagnostic tests against microscopy. Unlike in the previous studies where expert laboratory technicians interpreted the test results visually and operated the device, in this study low cadre health care workers who have not attended any formal professional training in laboratory sciences were employed.
\end{abstract}

Methods: Finger prick blood from 1293 outpatients with fever was tested for malaria using RDT and Giemsa-stained microscopy for thick and thin blood smears. Blood samples for RDTs were processed according to manufacturers' instructions automated in the Deki Reader. Results of malaria diagnoses were compared between visual and the automated devise reading of RDT and microscopy.

Results: The sensitivity of malaria rapid diagnostic test results interpreted by the Deki Reader was $94.1 \%$ and that of visual interpretation was $93.9 \%$. The specificity of malaria rapid diagnostic test results was $71.8 \%$ and that of human interpretation was $72.0 \%$. The positive predictive value of malaria RDT results by the Deki Reader and visual interpretation was 75.8 and $75.4 \%$, respectively, while the negative predictive values were 92.8 and $92.4 \%$, respectively. The accuracy of RDT as interpreted by DR and visually was 82.6 and $82.1 \%$, respectively.

Conclusion: There was no significant difference in performance of RDTs interpreted by either automated DR or visually by unskilled health workers. However, despite the similarities in performance parameters, the device has proven useful because it provides stepwise guidance on processing RDT, data transfer and reporting.

Keywords: Malaria, Automated, Deki Reader, RDT, Interpretation

\footnotetext{
*Correspondence: kalingaaka@yahoo.com

${ }^{1}$ National Institute for Medical Research, Tukuyu Centre, Tukuyu, Tanzania

Full list of author information is available at the end of the article
} 


\section{Background}

Malaria case management, consisting of early diagnosis and prompt effective treatment, remains a vital component of malaria control and elimination strategies [1]. Since 2010, it has been recommended by the World Health Organization (WHO) that all cases of suspected malaria should have a parasitological test by either quality microscopy or a rapid diagnostic test (RDT) to confirm the diagnosis before treatment can be administered [1]. Failure to confirm malaria using recommended diagnostic techniques would lead to using anti-malarial drugs to treat patients who do not have malaria infection and ultimately contributing to the development of drug resistance [2]. Moreover, overdiagnosis is not cost-effective because the currently used artemisinin-based combination therapy (ACT) drugs are expensive. Ultimately, the correct diagnosis reduces the costs of case management in the health system and provides an opportunity to diagnose and properly manage other malaria-like febrile conditions.

For a long time, good quality microscopy has been regarded as a gold standard method for diagnosis and confirmation of malaria parasite infections [2, 3]. However, in many settings, especially low resourced environments, laboratory confirmation by microscopy for case management is impractical due to unavailability of laboratory facilities including skilled laboratory microscopists at the point-of-care [4-6]. Moreover, good performance of microscopy can be difficult to maintain because it is dependent on adequate training [7] and supervision of laboratory staff, electricity, good quality of reagents, staining processes and slides and of quality assurance [1, 8]. Additionally, whenever microscopy is used fully or minimally by improperly trained technicians, the data obtained has been shown to be dubious and unreliable [9]. Thus, RDTs are being advocated and used as alternative, or as an adjunct to microscopy at health facilities because they can be easily used by health workers with less training and equipment, and can be performed by non-specialists in remote settings $[10,11]$. The RDTs provide fast test results for initiating or maintaining treatment of patients. However, for quality assurance, RDTs should be selected based on assessment results obtained using the WHO malaria RDT product testing programme $[12,13]$. In many malaria endemic areas RDTs are being used with more frequently along with microscopy or in place of microscopy [3]. Microscopy detects or quantifies parasitaemia per volume of blood (asexual parasites $/ \mu \mathrm{l}$ ). The accuracy of microscopy relies mostly on expertise of microscopists in preparation, staining and reading the results of blood slides. On the other hand, RDTs qualitatively detect the presence of circulating antigens in blood. Most of the available RDTs can detect three different types of Plasmodium antigens; Plasmodium histidine rich protein 2 (PfHRP-2), Plasmodium lactate dehydrogenase (Pf-pLDH) and Plasmodium aldolase (Pf-pAldo). For the case of PfHRP2 based RDTs, false positives are not uncommon because of persisting HRP2 antigens in the blood after several weeks of successful treatment of infections, while in Pf-pLDH based RDT, pLDH is glycolytic enzymes which is quickly removed following successful treatment as previously reported [14, 15].

Although fast and simple in concept, RDT performance in practice requires health workers to be trained on aspects of RDT test performance so that they are able to correctly interpret results and properly record them [16]. Considering its cost effectiveness for correct malaria diagnosis in primary health facilities as compared to other methods, RDT have been recommended as a tool for malaria confirmation and consequently have been shown to improve management and care of patients $[17,18]$. As such, there has been expansion of RDT usage worldwide which has aided management and care of malaria patients at health facilities of all levels [19]. For example, in 2013, approximately 319 million rapid diagnostic tests and 197 million slides were read and reported globally for the diagnosis and confirmation of malaria [20]. According to manufacturer sales data, RDTs sales increased from 46 million sold in 2008 to 314 million in 2014, and the number of diagnostic tests provided (RDTs and microscopy combined) exceeded the total number of courses of ACT administered in Africa [12].

In Tanzania, RDTs have become a solution for malaria diagnosis at rudimentary health care facilities such as dispensaries and health centres where microscopy is almost impossible [10]. Initially, RDTs were only used in research settings and this was followed by increased usage countrywide through a national roll-out campaign; from about two million in 2008 to 30 million RDTs in 2012 [21, 22]. Some common RDT brands that have been used in Tanzania include Paracheck $^{\circledR}$, ParaHIT, OptiMAL-IT ${ }^{\circledR}$, SD Bioline and CareStart ${ }^{\mathrm{TM}}$. The SD Bioline Malaria Antigen Pf/pan (Standard Diagnostics, Hagal-Dong, Korea), that detects PfHRP2 (Pf) and pLDH (pan), is the RDT that has been recommended by the Tanzanian National Malaria Control Programme (NMCP) for malaria diagnosis countrywide since 2012 [23]. The introduction of RDTs in Tanzania has radically improved malaria diagnosis to the extent that both overuse of anti-malarials and proper management of patients have been improved [24, 25]. For example, the proportion of suspected malaria cases tested by RDT and treated in public health facilities increased from 58\% in 2012 to $80 \%$ in 2016 [22].

Since RDTs are not able to detect parasitaemia infections at the lowest detection limit (usually $<100$ asexual parasites $/ \mu \mathrm{l}$ ) which is the parasitaemia level commonly 
observed in low malaria transmission settings, more sensitive detection methods are required to accurately detect malaria. At very low parasitaemia, the RDT test line is usually very weak and can be falsely interpreted as a negative test as reported in various studies conducted in Tanzania [24, 26, 27]. Thus, despite their apparent simplicity, the preparation and interpretation of RDTs can be suboptimal and poor interpretation of test results can impact patient care. End-users of RDTs are often health workers located in remote areas, often volunteers with limited training hence they are likely to make mistakes in both RDT test preparation and interpretation of results. To help address this problem, Fio Corporation (Toronto, Canada) designed a Deki Reader ${ }^{\text {TM }}$ (DR) to perform automated analysis and interpretation of RDTs. The DR is a ruggedized portable, universal reader of off-the-shelf lateral flow RDTs that provides a stable environment in which RDTs are imaged and results interpreted by onboard software. The device has been programmed to read most commonly used and commercially available histidine-rich-protein-2 (HRP2) based RDTs, including the SD Bioline malaria Ag Pf/pan (with both HRP2 and pLDH). Using a simple touch screen interface, an operator can enter patient identification number (ID), visually interpret RDT results, and input other patient information. This information is securely transmitted along with the RDT image and global positioning system (GPS) coordinates of the location to a centralized database over the local mobile phone network. All results from the field can be viewed from anywhere in the world in real-time by logging into a password protected portal using any web browser. Before validation of the DR for use in malaria detection, it was initially evaluated against traditional microscopy and polymerase chain reaction (PCR) in Colombia in South America and Tanzania in East Africa $[28,29]$. The initial evaluation was done by research institutions that used skilled laboratory technologists to operate the device for interpreting RDTs. However, under field use where skilled laboratory technologists are not available, the device will not be operated by professional laboratory personnel but rather by unskilled health care workers. These are low cadre health care workers who have not attended any formal professional training in laboratory sciences; they are also known as laboratory attendants/auxiliary. Initially, they were recruited as laboratory cleaners and through on-the-job training they became laboratory attendants to carry out simple laboratory tests like RDT. Therefore, prior to scaling-up the use of the device for routine medical care in Tanzania that is facing shortages of skilled health care workers [30]-a comparison of performance of RDT was made between RDT analysis done by DR that is operated by less skilled health workers and human visual interpretation of RDT results against microscopy as a reference test. The sensitivity, specificity, positive predicative value (PPV), negative predicative value (NPV) and accuracy of the RDT was compared between automated analysis by DR and visual interpretation.

\section{Methods \\ Study design and area}

This cross-sectional study was conducted at Mgambo health facility in Tanga region. Tanga region located in north eastern Tanzania is a malaria endemic area with two main malaria transmission seasons every year, the high malaria transmission seasons (HMTS) and low malaria transmission seasons (LMTS). The HMTS starts from April and runs through August, which is during and immediately after long rain season (April to June), and the LMTS starts from October and runs through December, which is the short rain season of the year. The study was conducted during the end of the rainy season (from June to July, 2014) during the high malaria transmission period in Tanga region.

\section{Study populations}

Study patients included military staff and civilians of all sexes and ages visiting the outpatient department at the military dispensary of Mgambo National Service Camp presenting with a fever (in the past $24 \mathrm{~h}$ ) likely attributable to malaria. All outpatients attended the facility during HMTS in 2014 were eligible to participate to the study.

\section{Recruitment of study participants}

Prior to recruitment, all outpatients or parents/guardian of sick children were informed about the study and requested to participate. They were asked for voluntary written informed consent prior to participation; two informed consent forms were filled by a participant or guardian and counter-signed by health personnel. One copy of the informed consent was kept within study file while the second copy was given to the participant. Thereafter, patients who gave informed consent were enrolled into the study.

\section{Training of data collectors}

Three military laboratory attendants volunteered for and trained for 2 days in the preparation and interpretation of RDTs per the guidelines of the NMCP in place at the time when this study was conducted. These health care workers were further trained for 2 days on how to use the DR for malaria diagnosis. Additionally, two laboratory assistants (who had attended 1 year laboratory foundation course) who were employees of Tanzania Peoples defense forces at National Service camp, were trained for 1 week 
on preparation of thick and thin blood smears, fixing of thin smears and staining of the smears.

\section{Malaria diagnosis with Deki Reader of RDTs}

In this study, 05fk60 SD Bioline Malaria Antigen Pf/pan (Standard Diagnostics Inc, Republic of Korea) RDT that detects PfHRP2 (P. falciparum) and pLDH (pan) was used for malaria diagnosis alongside microscopy. The DR provides automated sequential procedures to guide personnel on how to conduct the RDT test. The device prompts the user to insert the labeled RDT in a drawer for taking pictures of the RDT to recognize patient identification (ID) numbers before the test; thus, no patient names were entered in the device. After capturing an image and recognizing the brand of the RDT, the device prompts the user to remove the RDT from the device to proceed with taking blood samples from the patient. About $5 \mu \mathrm{l}$ of blood sample was collected from a finger prick using a standard sampler (provided by the RDT manufacturer) and added to the blood well of the RDT cassette. Four drops of buffer were immediately added to the second well of the RDT cassette. After the incubation period (27 min for SD Bioline malaria Ag Pf/pan complying with manufacturer guidelines) as guided by the automated timer in the device, the user was prompted to insert the RDT in the device for the second time to capture an image of the RDT test which was then analysed and interpreted by the DR. The user was also prompted to input his/her own visual interpretation of the test results for both assays, i.e. P. falciparum and the panmalaria line. The test results interpreted by the DR were not displayed on the device because the user was masked to the DR results. Both visual and device interpretation test results along with the image of the RDT and patient de-identified bio data were directly uploaded to the study web-portal.

\section{Malaria diagnosis using microscopy}

From the same finger prick, thick and thin blood smears were prepared as guided by a standardized template; a slide with one frosted end was placed on top of the template and $6 \mu \mathrm{l}$ of blood was immediately added to the slide on a large circle marked on the template which was then spread to cover the circle. Similarly, $2 \mu$ l blood was added at the edge of a small circle of the template for the thin film, which was then smeared as per standard operating procedures [31]. The prepared blood smears were air-dried, the thin smears were fixed by immersing in $100 \mathrm{ml}$ beaker full of methanol Analar ${ }^{\circledR}$, and both smears (thick and thin) were stained using 10\% working solution of fresh Giemsa for $30 \mathrm{~min}$. The stained smears were examined by experienced senior microscopists at Tanga Research Centre of the National Institute for Medical
Research (NIMR) who was blinded of the results of the RDT interpreted visually and by the DR. To confirm positive films, asexual parasites were counted against 200 white blood cells (WBCs) while sexual parasites were counted against 500 WBCs. Assuming that each microlitre $(\mu \mathrm{l})$ of blood contains 8000 WBCs parasites, densities were calculated by multiplying the parasite counts by 40 for asexual and 16 for sexual parasites. A smear was regarded to be negative after examining 200 highpower fields and not detecting parasites. Malaria species were detected and differentiated using thin blood smears depending on the appearance of the parasite, size and shape of the surrounding red cells, the presence of dots, stippling and pigment [31]. Other characteristics used for differentiation of the species included appearance of gametocytes and the developmental stages seen.

\section{Data management and analysis}

Microscopy data were entered into Microsoft Access Data base. The data were double entered by two different data clerks, checked for consistency, validated and cleaned. Each patient's record was identified by unique identification number which later was used to link microscopic data with patient's de-identified demographic information and RDT results downloaded from secured web-portal. Data from RDT and the patients' demographic information were entered by touching the screen interface in the DR and uploaded through mobile networks to a secured web-portal. The web-portal was accessed only by authorized project staff using password-protected. Data from RDT were downloaded to Microsoft Excel, checked, cleaned and then transferred to STATA software (Stata Corp, College Station, TX) for analysis. This data was merged with microscopy results, whereby all mismatched or uncorrected data were removed, including dropping of duplicate data to prepare the master file. Descriptive statistics with mean, standard deviations and proportions were used to summarize demographic information of the study participants Chi square was used to assess the associations between categorical variables. The sensitivity, specificity, positive predictive value (PPV), and negative predictive value (NPV) was used to compare automated RDT analysis and visual human interpretation against microscopy as reference test. A contingency table was used to calculate the indices where sensitivity as $\mathrm{TP} / \mathrm{TP}+\mathrm{FN}$, specificity as TN/TN+FP, PPV as TP/TP+FP, NPV as TN/TN+FN and accuracy of the test as $\mathrm{TP}+\mathrm{TN} / \mathrm{N}$. In these formulas $\mathrm{N}=$ number of individuals tested; $\mathrm{TP}=$ true positive $(\%$ of those who tested positive by both RDTs and microscopy; $\mathrm{TN}=$ true negative (\% of those tested negative by RDTs and microscopy; $\mathrm{FN}=$ false negative (\% of those who tested falsely as negative by RDTs while they actually 
were positive by microscopy; $\mathrm{FP}=$ false positive (\% of those who tested falsely as positive by RDTs while they actually were negative by microscopy). The sensitivity was presented as proportion with $95 \%$ confidence intervals. $\mathrm{P}$-value was considered significant at $\mathrm{P}<0.05$.

\section{Results}

A total of 1293 among 1423 outpatients with axillary temperature $\geq 37.5{ }^{\circ} \mathrm{C}$, reported fever in the past $24 \mathrm{~h}$ and/or other symptoms suggestive of malaria were recruited in the study. A total of 130 patients did not participate in this study of which 30 did not consent, 64 had no blood smears prepared together with RDTs and 36

Table 1 Demographics of participants and baseline diagnostic characteristics

\begin{tabular}{|c|c|}
\hline Variable & \\
\hline Number of participants, $\mathrm{n}$ & 1293 \\
\hline \multicolumn{2}{|l|}{ Sex, n (\%) } \\
\hline Male & $893(69.1)$ \\
\hline Female & $400(30.9)$ \\
\hline \multicolumn{2}{|l|}{ Age (years) } \\
\hline Median (IQR) & $21(20-23)$ \\
\hline \multicolumn{2}{|l|}{ Age group (years), $n(\%)$} \\
\hline $0-17$ & $139(10.8)$ \\
\hline $18-24$ & $923(71.4)$ \\
\hline $25+$ & $231(17.9)$ \\
\hline \multicolumn{2}{|l|}{ RDT positivity rates $\mathrm{n}(\%)$} \\
\hline By Deki Reader & $777(60.1)$ \\
\hline Visual interpretation & $769(59.9)$ \\
\hline \multicolumn{2}{|l|}{ Parasitaemia infection } \\
\hline Microscopy positivity rate, $\mathrm{n}(\%)[95 \% \mathrm{Cl}]$ & $48.4(45.7-51.2)$ \\
\hline Parasite density range (asexual parasites/ $\mu$ l) & $0-205,120$ \\
\hline GMPD [asexual parasites/ $\mu$ l (95\% Cl] & $3399(2872-4022)$ \\
\hline
\end{tabular}

$\mathrm{Cl}$, confidence interval at 95\%; IQR, interquartile range; GMPD, geometric mean parasite density had smears of low quality to carry out further analysis. The median age for study participants was 21 (Interquartile range 21-23) years (Table 1) and the majority of the participants $(71.4 \%$; 923/1293) were aged $18-24$ years. More than two third $(69.1 \%$; 893/1293) of study participants were male.

Malaria RDT data were uploaded to portal by DR at speed of $\geq 76 \%$ of the records for a period of $\leq 24 \mathrm{~h}$ and only $26 \%$ of data were uploaded between 1 and 7 days. Malaria positivity rates by RDT were $60.1 \%$ (777/1293) and $59.9 \%(775 / 1293)$ as interpreted by DR and visually, respectively. Malaria positivity rate by microscopy as a reference test was $48.4 \%(626 / 1293)$ with a geometric mean parasite density of 3399 (95\% CI $=2872-4022)$ asexual parasites/ $\mu \mathrm{l}$ (Table 1 ).

Plasmodium falciparum was predominant species constituting $99.7 \%(624 / 626)$ of all malaria species found and two samples were mixed infections of $P$. falciparum and Plasmodium malariae (Table 2). Out of the 624 smearpositive $P$. falciparum infection samples, 5.8\% (36/624) were falsely diagnosed as negative by automated DR and $6.4 \%$ (40/624) by visual interpretation (Table 3). Among the falsely diagnosed negative samples $5.0 \%(31 / 624)$ of all positives were the same samples diagnosed by both DR and visual interpretation. Out of two samples of mixed infections (P. falciparum and P. malariae), in one smear positive sample $P$. malariae was falsely diagnosed as negative by both DR and visual interpretation. Out of the 667 smear negative samples, $28.2 \%(188 / 667)$ were falsely diagnosed as positive by DR and $27.6 \%(184 / 667)$ by visual interpretation (Table 3 ).

In reference to microscopy test as a gold standard, the sensitivity of RDTs in malaria diagnosis as interpreted by the device was $94.1 \%$ (95\% CI 91.8-95.7) and that of human visual interpretation of RDTs was $93.5 \%$ (95\% CI 91.6-95.5). The specificity of RDTs in malaria diagnosis as interpreted by the device was $71.8 \%$ (95\% CI 67.3-74.3) and that of human visual interpretation of

Table 2 Characterization of malaria plasmodium parasite species and antigens

\begin{tabular}{|c|c|c|c|c|c|c|c|c|c|c|c|}
\hline \multirow{2}{*}{\multicolumn{2}{|c|}{ Microscopy +ve }} & \multicolumn{5}{|c|}{ Deki Reader analysis } & \multicolumn{5}{|c|}{ Visual interpretation } \\
\hline & & \multicolumn{4}{|l|}{ TP } & \multirow[t]{2}{*}{ FN } & \multicolumn{4}{|l|}{ TP } & \multirow[t]{2}{*}{ FN } \\
\hline Species & No. (\%) & Pan & Pf & Pf and pan & All & & Pan & Pf & Pf and pan & All & \\
\hline Pf & $624(48.3)$ & 5 & 285 & 298 & $588(93.9)$ & $36(5.8)$ & 3 & 287 & 294 & $584(93.6)$ & $40(6.4)$ \\
\hline Po & $0(0)$ & 0 & 0 & 0 & $0(0)$ & $0(0)$ & 0 & 0 & 0 & $0(0)$ & $0(0)$ \\
\hline $\mathrm{Pm}$ & $0(0)$ & 0 & 0 & 0 & $0(0)$ & $0(0)$ & 0 & 0 & 0 & $0(0)$ & $0(0)$ \\
\hline $\mathrm{Pf}+\mathrm{Pm}$ & $2(0.2)$ & 0 & 1 & 0 & $1(50.0)$ & $1(50.0)$ & 0 & 1 & 0 & $1(50.0)$ & $1(50.0)$ \\
\hline$P f+P o$ & $0(0)$ & 0 & 0 & 0 & $0(0)$ & $0(0)$ & 0 & 0 & 0 & $0(0)$ & $0(0)$ \\
\hline All & $626(48.4)$ & 5 & 286 & 298 & $589(94.1)$ & $37(5.9)$ & 3 & 288 & 294 & 585 (93.5) & $41(6.5)$ \\
\hline
\end{tabular}

PT, true positive; FN, false negative; Pf, P. falciparum; Pm, P. malariae; $\mathrm{Po}$, P. ovale 
Table 3 Cross tabulation of RDT with microscopy test results, and performance of the RDT

\begin{tabular}{|c|c|c|c|c|}
\hline \multirow[t]{2}{*}{ RDT (any band) } & \multicolumn{2}{|c|}{ Microscopy } & \multirow[t]{2}{*}{ Total } & \multirow[t]{2}{*}{ RDT performance } \\
\hline & Positive & Negative & & \\
\hline \multicolumn{5}{|l|}{ DR analysis } \\
\hline Positive & 589 & 188 & 777 & Sensitivity $=589 / 626 * 100=94.1 \%$ \\
\hline Negative & 37 & 479 & 516 & Specificity $=479 / 667 * 100=71.8 \%$ \\
\hline \multirow[t]{2}{*}{ Total } & 626 & 667 & 1293 & $P P V=589 / 777 * 100=75.8 \%$ \\
\hline & & & & $N P V=479 / 516^{*} 100=92.8 \%$ \\
\hline \multicolumn{5}{|c|}{ Visual interpretation } \\
\hline Positive & 585 & 184 & 769 & Sensitivity $=585 / 626 * 100=93.5 \%$ \\
\hline Negative & 41 & 483 & 524 & Specificity $=483 / 667 * 100=72.4 \%$ \\
\hline \multirow[t]{2}{*}{ Total } & 626 & 667 & 1293 & $P P V=585 / 769 * 100=76.1 \%$ \\
\hline & & & & $N P V=483 / 524 * 100=92.2 \%$ \\
\hline
\end{tabular}

DR, Deki Reader; PPV, positive predictive value; NPV, negative predictive value

Table 4 Comparison of sensitivity of RDT between automated analysis by Deki Reader and visual interpretation at different levels of parasite density

\begin{tabular}{lll}
\hline & \multicolumn{2}{c}{ Sensitivity of RDT; $\mathbf{n} / \mathbf{N}(\%)$} \\
\cline { 2 - 3 } & $\begin{array}{c}\text { Deki Reader } \\
\text { interpretation }\end{array}$ & Visual interpretation \\
\hline Parasite density (asexual parasites/ $\mu$ l) & \\
$\geq 4001$ & $325 / 327(99.4)$ & $325 / 327(99.4)$ \\
$2001-4000$ & $67 / 67(100)$ & $67 / 67(100)$ \\
$801-2000$ & $62 / 65(95.4)$ & $62 / 65(95.4)$ \\
$201-800$ & $72 / 76(94.7)$ & $71 / 76(93.4)$ \\
$<200$ & $63 / 91(69.2)$ & $63 / 91(69.2)$ \\
Statistical tests & & \\
Chi square & 122.1 & 119.1 \\
P-value & 0.000 & 0.000 \\
\hline
\end{tabular}

RDTs was 72.4\% (95\% CI 67.3-74.3). The PPV of RDT interpreted by the DR and human was $75.8 \%$ (95\% CI 72.0-78.2) and 76.1\% (95\% CI 72.3-78.4), respectively. The NPV of RDT interpreted by the DR and interpreted visually were $92.8 \%$ (95\% CI 89.9-94.7) and $92.2 \%$ (95\% CI 89.8-94.6), respectively. The accuracy of RDT as interpreted by DR and visually was 82.6 and $82.1 \%$, respectively. There was no significance difference between the PPV, NPV, the accuracy of RDT interpretation by automated DR and visual interpretation by a semi-skilled laboratory staff.

Table 4 shows that an increase in parasite density from $<200$ parasites/ $\mu \mathrm{l}$ to 4000 parasites/ $\mu \mathrm{l}$ ) was associated with increased sensitivity of RDT, from 69.2 to $100 \%$, respectively; and the difference was statistically significant $(\mathrm{P}=0.000)$. The trends were similar within and between RDT interpreted visually by human and by automated analysis.

\section{Discussion}

In this study, automated analysis by DR and visual interpretation of RDTs by unskilled health care workers against microscopy was compared. This comparative study in interpreting RDTs between unskilled cadre of health workers and automated DR for routine outpatients is the first to be done in rural military health facilities. Whereas previous studies conducted in Tanzania, Colombia and Uganda [28, 29, 32] employed laboratory technicians from research institutions in performing RDT testing and operating the DR, in this study unskilled health care workers who had no formal training in laboratory sciences were employed to operate the automated device and also to visually interpret RDT test results. In the current trend of expanding the use of RDTs to the community level, community health workers are becoming solely responsible for malaria testing. Because of this, unskilled laboratory staff were chosen to operate the DR and visually interpret the RDT tests for comparative analysis.

In this study, the sensitivity, specificity, PPV, NPV, and accuracy RDTs as interpreted by the automated DR (94.1, $71.8,75.8$ and $82.6 \%$ ) were similar to the interpretations which were visually done by unskilled health care workers $(93.5,72.4,76.1$ and $82.1 \%)$ with no significant differences. These findings are consistent with the previous studies conducted in Colombia, Tanzania and Uganda $[28,29,32]$ where the interpretations of RDTs by automated device were comparable to expert visual interpretation (concordance rate $>95 \%$ ) for $P$. falciparum which is the dominant malaria species in Tanzania.

Comparing this study with other recent studies that did not use DR for interpretation of test results in Tanzania, in this study the sensitivity of HRP2-based RDTs of about $94 \%$ which is lower than the $>95 \%$ recommended by the 
WHO [1]. Two studies conducted in Zanzibar, a constituent state of the United Republic of Tanzania observed a lower sensitivities of $79 \%$ [33] and $78.6 \%$ and higher specificity of $99.7 \%$ [33] compared to that reported in this study. Other studies done in Korogwe-Tanga of Tanzania [24, 34] also reported lower sensitivities of 88.6 and $88.9 \%$ and higher specificity of 88.2 and $97.4 \%$ respectively, compared to findings of this study. In 2008, in Rufiji and in Korogwe, investigators observed a sensitivity as low as $65 \%$ [35] and $88.9 \%$ [34], respectively. The sensitivity from this study (94.0\%) is slightly lower than that reported in a study done in 2016 at multiple Tanzanian sites in Muheza, Ujiji, Muleba and Nachingwea where they observed sensitivity ranging from 97.3 to $99.3 \%$ at all sites [36] . A relative higher sensitivity (>90\%) for RDTs interpreted by both device and human coupled with high NPV in this study, is an indicative that the test is good for malaria diagnosis at point of care because very few patients will be missed by the test. Unfortunately in this study only one batch of RDT was used; it will be useful to test different batches in future studies to compare the performance of RDT for each batch.

The observed high sensitivity and low specificity of RDT should have been affected by high malaria transmission in the study area because high transmission is characterized by high false positive results and hence high sensitivity and low specificity as reported elsewhere [24]. In this regard and corresponding to late 2000s and early 2010s, when malaria transmission declined noticeably, the sensitivity of RDTs was low, but recently over the past 2 years, higher malaria transmission rates and parasite densities have been observed in Tanzania [36] and the sensitivity of RDTs has been observed to be a bit higher as reported in the recent studies $[34,36]$.

The reported specificity of about $72 \%$ found in this study is lower compared to what has been reported in other studies presumably due to the reported higher false positivity rate (28\%), which could be due to persistence of PfHRP2 in the blood stream for several weeks after successful treatment leading clearance of parasites as reported elsewhere [15, 37]. Moreover, this study population constituted mostly (72\%) by military recruits who were mostly coming from low malaria endemic areas and hence susceptible to infection in the study area which is highly endemic to malaria transmission. The high malaria prevalence in the area as demonstrated through high positivity rate by microscopy should have contributed to low specificity and high sensitivity of RDT as reported elsewhere [37, 38].

The sensitivity of RDT interpreted by automated DR and visually had a direct relationship with parasite density. The lower the parasite density ( $\leq 200$ asexual parasites/ $\mu \mathrm{l}$ ) among blood samples, the lower the sensitivity of the RDT interpreted by the device (69.2\%) and visually (69.2\%), while the higher the parasite density (2001-4000 asexual parasites/ $\mu \mathrm{l}$ ) among blood samples, the higher the sensitivity $(100 \%)$ of the RDT interpreted by the device and visually. At the highest parasite density level ( $\geq 4001$ asexual parasites/ $\mu \mathrm{l})$, higher sensitivity $(99.4 \%)$ was also registered although slightly lower by $0.6 \%$ compared to the maximum (100\%) detection limit. This finding is in concordance with previous studies done in Tanzania [24,39] that reported the direct proportionality of parasite density and prevalence with HRP2-based RDT. Other studies done in Nigeria [40] and Korea [41] also found that the sensitivity of RDT increased significantly $(\mathrm{P}<0.0001)$ with an increase in $P$. falciparum parasitaemia. In contrary, in Central African Republic, although the tests performed less well in cases of low parasitaemia, they reported sensitivity of $>95 \%$ at $>500$ parasites/ $\mu$ l [42] as opposed to sensitivity in this study (94.7\%) at 201-800 parasites/ $\mu \mathrm{l}$. The sensitivity of RDT in relation to parasite densities as reported in this study samples are comparable to previous studies that reported in standardized categories of parasite density ranging from lower density ( $\leq 200$ asexual parasites/ $\mu$ l) to higher density ( $\geq 2000$ asexual parasites/ $\mu \mathrm{l}$ ) [43].

In this study, a false positivity rate of $28 \%$ was found by RDTs as interpreted by both the device and visually by unskilled health care workers. Such a high discordance rate could be attributed to the presence of persistent malaria antigens in the blood due to unreported recent self or hospital treatment with anti-malarials and hence leading to false positives which, has also been reported in other studies that used HRP2-based RDTs [44, 45]. The results of this study showed that $\leq 0.8 \%(5 / 626)$ of the samples were only detected by pan, while about $46 \%$ (286/626) were detected by Pf and $\geq 47 \%(298 / 626)$ by both Pf and Pan. Since pLDH detects both Pf and nonfalciparum species, these results show that an RDT without pLDH would have missed less than $0.8 \%$ of the samples while pLDH missed over $45 \%$ of the samples, which were detected by the Pf band only. But since pLDH tests are usually combined with HRP2, these findings suggest that a combo test like SD Bioline is unlikely to miss significant number of parasites given the high sensitivity reported in this study.

In this regard, high false-positivity rate accompanied with low specificity can result into over diagnosis of malaria among non-malaria febrile patients. If a patient is found to be positive by HRP2 RDT simply due to history of recent malaria treatment, that result should be investigated more thoroughly and other possible causes of non-malaria fever should be investigated for proper treatment. However, the reported high false positive rate can be valid only if microscopy was performed very 
accurately (taking into account that PCR was not performed to correct microscopy results), otherwise, some reported false results in this study could be correct diagnosis as also reported in recent studies that RDTs are becoming more sensitive than microscopy performed inaccurately especially in low malaria transmission settings $[46,47]$. Additionally, patients detected positive by RDT could have pure gametocytaemia that cannot be detected microscopically as studied elsewhere that presence of antigen secreting gametocytes is another possible cause of persistent HRP2 or pLDH antigenaemia [48]. Whilst, about $6 \%$ false negativity rate that was obtained could be due to deletion of $p f h r p 2$ or/and $p f h r p 3$ gene as also reported by other studies [49-51]. The reported false negative rate in this study could be also attributed by prozone effects as also reported by other studies [49]. In this study, it was observed that the weak test line on RDT sometimes could not be visualized by laboratory personnel with a decline in visual acuity and poor lighting systems in the testing room of these remote poor resource settings.

\section{Study limitations}

Unlike other studies that employed polymerase chain reaction (PCR) for resolving discordant results of RDTs compared to that of microscopy, this study only used microscopy to confirm RDT results. Thus, dependence on microscopy to confirm RDT test results may have impacted these results because microscopy test results are likely to be affected by transmission intensity, prevalence of infections and parasite density as reported elsewhere [1].

\section{Conclusion}

The performance agreement of the DR compared to visual interpretation conducted by unskilled health care workers in interpreting RDTs does suggest that the device can be used alone or concurrently with microscopy for malaria diagnosis. The use of this DR device at the point of care not only interprets RDT results, but has the added advantage to provide stepwise procedures for RDT testing, data transfer and timely reporting as also studied in Kenya and Uganda [32, 52]. Moreover, similar to skilled laboratory technologists, unskilled laboratory staff can be trained how to operate the DR for couple of days, assigned and use it for malaria diagnosis in remote areas, such as military camps; the DR is mostly automated in its operation and is, therefore, simple to use by any laboratory staff regardless level of education or professional training in laboratory technology. Therefore, it can be concluded that automated analysis of RDT by the DR operated by unskilled health care workers was similar to visual interpretation of RDT, but it has the added benefit by timely collecting surveillance data from rural settings and allowing managers to review RDT results from multiple rural sites simultaneously.

\section{Authors' contributions}

AK, CO, GB and IF designed the study; LM, GA, LT, CMS and SM supervised Laboratory diagnosis field work and contributed to draft the manuscript. FF, $\mathrm{NZ}, \mathrm{EK}$ and DI analysed data and contributed to drafts of the manuscript. CMZ, LA, EW, SC and LT drafted the manuscript. RK, DI, BV, RP, KD, SF, MH, NW and $\mathrm{CO}$ revised the manuscript. AK was responsible for writing and coordination of drafting the manuscript. All authors read and approved the final manuscript.

\section{Author details \\ ${ }^{1}$ National Institute for Medical Research, Tukuyu Centre, Tukuyu, Tanzania. ${ }^{2}$ Henry Jackson Foundation Medical Research International, Dar es Salaam, Tanzania. ${ }^{3}$ Tanzania Peoples Defense Forces, Dar es Salaam, Tanzania. ${ }^{4}$ National Institute for Medical Research, Tanga Centre, Tanga, Tanzania. ${ }^{5}$ Wal- ter Reed Army Institute of Research, 503 Robert Grant Ave, Washington DC, USA. ${ }^{6}$ Fio Corporation, Toronto, Canada. ${ }^{7}$ FORGYN Health Systems Consult- ants LLC, Silver Spring, USA. ${ }^{8}$ Kilimanjaro Christian Medical University College, Moshi, Tanzania. ${ }^{9}$ Consortium for Health Action, Phnom Penh, Cambodia.}

\section{Acknowledgements}

We thank the Tanzania Peoples Defense Forces (TPDF), the National institute for Medical Research (NIMR), the Commission for Science and Technology (COSTECH) in Tanzania, the Fio Corporation in Canada, the Walter Reed Army Institute of Research (WRAIR), the Henry Jackson Foundation Medical Research Institute (HJFMRI), Clinical Research Malaria (CRM) Inc, FORGYN Health Systems Consultants LLC and Amethyst Technologies Limited (ATL) in the US for their financial and management support to successfully conduct this study. Special thanks are extended to all laboratory personnel who participated in this study conducting malaria diagnosis using RDTs at the point of care and microscopists at the centralized research laboratory at NIMR-Tanga. Finally, we are grateful to patients who enrolled as study participants at Mgambo military dispensary.

\section{Competing interests}

The authors declare that they have no competing interests. However, four authors namely SF, IF, GB and NZ are employees or consultants of Fio Corporation, the creator of the DR device.

\section{Availability of data and materials}

The datasets analysed during the current study are available from the corresponding author, office of Chief of Medical Services of Peoples defense forces or/and Walter Reed malaria programme on reasonable request.

\section{Consent for publication}

Not applicable.

\section{Ethical approval and consent to participate}

This study was approved by the National Health Review Committee (NatHREC) of the national Medical Research Coordinating Committee (certificate \# NIMR/ HQs/R.8a/Nol. IX/1338) and Kilimanjaro Christian Medical University College's (KCMUCo) Research Ethics Review Committee (CRERC \# 818) in Tanzania. Permission to conduct the study in military health facilities was sought from the Chief of Medical Services at the headquarters of the Tanzania Peoples Defense Forces (TPDF) as well as the Commanding Officers (COs) of the respective military camps where the health facility was located. Patients were asked for voluntary written informed consent prior to participation; two informed consent forms were filled by a participant or guardian and counter-signed by health personnel. One copy of the informed consent was kept within study file while the second copy was given to the participant. Patients diagnosed positive for malaria using RDT interpreted visually were treated as per malaria treatment guidelines $[22,52]$

\section{Funding}

The conduct of this study was enabled through funding received from Walter Reed Army Institute of Research (WRAIR) for malaria surveillance and diagnostic quality improvement programme in Tanzania. Material has been reviewed by the Walter Reed Army Institute of Research. There is no objection to its 
presentation and/or publication. The opinions or assertions contained herein are the private views of the author, and are not to be construed as official, or as reflecting true views of the Department of the Army or the Department of Defense. The investigators have adhered to the policies for protection of human subjects as prescribed in AR 70-25.

\section{Publisher's Note}

Springer Nature remains neutral with regard to jurisdictional claims in published maps and institutional affiliations.

Received: 1 March 2018 Accepted: 22 May 2018

Published online: 29 May 2018

\section{References}

1. WHO. Guidelines for the treatment of malaria. 3rd ed. Geneva: World Health Organization; 2015.

2. WHO. World malaria report 2015. Geneva: World Health Organization; 2015. WHO/HTM/GM:243. http://www.who.int/malaria/publications/ world_malaria_report_2013/en/\%5Cnhttp://www.who.int/iris/bitst ream/10665/97008/1/9789241564694_eng.pdf\%5Cnhttp://www.who. int/malaria/publications/world_malaria_report_2013/wmr2013_no_profi les.pdf?ua=1. Accessed 27 Apr 2017.

3. Wongsrichanalai C, Barcus MJ, Muth S, Sutamihardja A, Wernsdorfer WH. A review of malaria diagnostic tools: microscopy and rapid diagnostic test (RDT). Am J Trop Med Hyg. 2007;77:119-27.

4. Hamer DH, Ndhlovu M, Zurovac D, Fox M, Yeboah-Antwi K, Chanda P, et al. Improved diagnostic testing and malaria treatment practices in Zambia. JAMA. 2007;23:2227-31

5. Petti CA, Polage CR, Quinn TC, Ronald AR, Sande MA. Laboratory medicine in Africa: a barrier to effective health care. Clin Infect Dis. 2006;42:377-82.

6. Mboera LEG, Makundi EA, Kitua AY. Uncertainty in malaria control in Tanzania: crossroads and challenges for future interventions. Am J Trop Med Hyg. 2007;77:112-8.

7. Moura S, Fançony C, Mirante C, Neves M, Bernardino L, Fortes F, et al. Impact of a training course on the quality of malaria diagnosis by microscopy in Angola. Malar J. 2014;13:347

8. Font F, Alonso Gonzalez M, Nathan R, Kimario J, Lwilla F, Ascaso C, et al. Diagnostic accuracy and case management of clinical malaria in the primary health services of a rural area in south-eastern Tanzania. Trop Med Int Health. 2001;6:423-8.

9. Kahama-Maro J, D'Acremont V, Mtasiwa D, Genton B, Lengeler C. Low quality of routine microscopy for malaria at different levels of the health system in Dar es Salaam. Malar J. 2011;10:332.

10. Programme National Malaria Control. National guidelines for diagnosis and treatment of malaria. Dar es Salaam: National Malaria Control Programme; 2006. p. 1-105.

11. Visser T, Daily J, Hotte N, Dolkart C, Cunningham J, Yadav P. Rapid diagnostic tests for malaria. Policy and practice. Bull World Health Organ. 2015;93:862-6.

12. WHO/FIND/CDC. Malaria rapid diagnostic test performance: Summary results of WHO product testing of malaria RDTs: rounds 1-6 (2008-2015). 2015. http://www.finddx.org/wp-content/uploads/2016/04/summaryresults-malaria_rdts_rounds1-6-2008-2015.pdf. Accessed 3 Oct 2017.

13. WHO. Global malaria programme information note on recommended selection criteria for procurement of malaria rapid diagnostic tests (RDTs). Geneva: World Health Organization; 2012.

14. Gerstl S, Dunkley S, Mukhtar A, De Smet M, Baker S, Maikere J. Assessment of two malaria rapid diagnostic tests, with followup of positive pLDH test results, in a hyperendemic falciparum malaria area. Trop Med Int Health. 2009;14:92.

15. Singh N, Shukla MM. Field evaluation of posttreatment sensitivity for monitoring parasite clearance of Plasmodium falciparum malaria by use of the Determine ${ }^{\mathrm{TM}}$ Malaria pf test in central India. Am J Trop Med Hyg 2002;66:314-6.

16. Bell D, Wongsrichanalai C, Barnwell JW. Ensuring quality and access for malaria diagnosis: how can it be achieved? Nat Rev Microbiol. 2006;4:682-95.
17. Msellem MI, Mårtensson A, Rotllant G, Bhattarai A, Strömberg J, Kahigwa $E$, et al. Influence of rapid malaria diagnostic tests on treatment and health outcome in fever patients, Zanzibar - a crossover validation study. PLoS Med. 2009;6:e10000070.

18. Chanda P, Castillo-Riquelme M, Masiye F. Cost-effectiveness analysis of the available strategies for diagnosing malaria in outpatient clinics in Zambia. Cost Eff Resour Alloc. 2009;7:5.

19. Drakeley C, Reyburn H. Out with the old, in with the new: the utility of rapid diagnostic tests for malaria diagnosis in Africa. Trans $\mathrm{R}$ Soc Trop Med Hyg. 2009;103:333-7.

20. WHO. World malaria report 2014. Geneva: World Health Organization; 2014.

21. Tanzania Commission for AIDS (ZAC), Zanzibar AIDS Commission (NBS), National Bureau of Statistics (OCGS) O of the CGSII. Tanzania-2011-2012 HIV/AIDS and Malaria Indicator Survey 2011-2012: Key Findings. 2013.

22. National Malaria Control Programme. National malaria strategic plan 2014-2020. Dar es Salaam. 2014. http://ihi.eprints.org/3314/1/Malar ia_Strategic_Plan_Full_Version_02_27_14.pdf. Accessed 16 Jan 2018.

23. Tanzania Commission for AIDS (TACAIDS), Zanzibar AIDS Commission (ZAC), National Bureau of Statistics (NBS), Office of the Chief Government Statistician (OCGS), and ICF International. Tanzania HIV/AIDS and Malaria Indicator Survey 2011-2012: Key Findings. Da and II. Tanzania 2011-2012 HIV/AIDS and malaria Indicator Survey: Key Findings. 2013.

24. Ishengoma DS, Francis F, Mmbando BP, Lusingu JPA, Magistrado P, Alifrangis $M$, et al. Accuracy of malaria rapid diagnostic tests in community studies and their impact on treatment of malaria in an area with declining malaria burden in north-eastern Tanzania. Malar J. 2011:10:176.

25. Rutta ASM, Francis F, Mmbando BP, Ishengoma DS, Sembuche SH, Malecela EK, et al. Using community-owned resource persons to provide early diagnosis and treatment and estimate malaria burden at community level in north-eastern Tanzania. Malar J. 2012;11:152.

26. Buhalata SN, Massaga JJ. Performance of ParaHit and OptiMAL tests in the diagnosis of malaria in Mwanza, north-western Tanzania. Tanzan J Health Res. 2011;13:48-53.

27. Manjurano A, Okell L, Lukindo T, Reyburn H, Olomi R, Roper C, et al. Association of sub-microscopic malaria parasite carriage with transmission intensity in north-eastern Tanzania. Malar J. 2011;10:370.

28. Herrera S, Vallejo AF, Quintero JP, Arévalo-Herrera M, Cancino M, Ferro $\mathrm{S}$. Field evaluation of an automated RDT reader and data management device for Plasmodium falciparum/Plasmodium vivax malaria in endemic areas of Colombia. Malar J. 2014;13:87.

29. Shekalaghe S, Cancino M, Mavere C, Juma O, Mohammed A, Abdulla S, et al. Clinical performance of an automated reader in interpreting malaria rapid diagnostic tests in Tanzania. Malar J. 2013;12:141.

30. Mcharo J, Munga MA, Mushi AK, Mtui T, Kilale A, Makamba Y, et al. Taskshifting in the provision of HIV/AIDS, Reproductive and Child Health $(\mathrm{RCH})$ Services in Tanzania: exploring epidemiology the views and experiences of health managers. SM J Public Health Epidemiol. 2016;2:1034.

31. WHO. Basic malaria microscopy: Part 1. Learner's guide. 2nd ed. Geneva: World Health Organization; 2010.

32. Oyet C, Roh ME, Kiwanuka GN, Orikiriza P, Wade M, Parikh S, et al. Evaluation of the Deki Reader ${ }^{\mathrm{rM}}$, an automated RDT reader and data management device, in a household survey setting in low malaria endemic southwestern Uganda. Malar J. 2017;16:449.

33. Shakely D, Elfving K, Aydin-Schmidt B, Msellem MI, Morris U, Omar R, et al. The usefulness of rapid diagnostic tests in the new context of low malaria transmission in Zanzibar. PLoS One. 2013;8:e72912.

34. Mahende C, Ngasala B, Lusingu J, Yong T-S, Lushino P, Lemnge M, et al. Performance of rapid diagnostic test, blood-film microscopy and PCR for the diagnosis of malaria infection among febrile children from Korogwe District, Tanzania. Malar J. 2016;15:391.

35. McMorrow ML, Masanja MI, Abdulla SMK, Kahigwa E, Kachur SP Challenges in routine implementation and quality control of rapid diagnostic tests for malaria-Rufiji District, Tanzania. Am J Trop Med Hyg. 2008;79:385-90.

36. Ishengoma D, Shayo A, Ci M, Baraka $\vee$, Ra M, Ngatunga $D$, et al. The role of malaria rapid diagnostic tests in screening of patients to be enrolled in clinical trials in low malaria transmission settings. Health Syst Policy Res. 2016;3:2.

37. Maltha J, Guiraud I, Lompo P, Kaboré B, Gillet P, Van Geet C, et al. Accuracy of PfHRP2 versus Pf-pLDH antigen detection by malaria rapid diagnostic 
tests in hospitalized children in a seasonal hyperendemic malaria transmission area in Burkina Faso. Malar J. 2014;13:20.

38. Mtove G, Hendriksen IC, Amos B, Mrema H, Mandia V, Manjurano A, et al. Treatment guided by rapid diagnostic tests for malaria in Tanzanian children: safety and alternative bacterial diagnoses. Malar J. 2011;10:290

39. Mmbando BP, Vestergaard LS, Kitua AY, Lemnge MM, Theander TG, Lusingu JPA. A progressive declining in the burden of malaria in northeastern Tanzania. Malar J. 2010;9:216.

40. Oyeyemi OT, Ogunlade AF, Oyewole IO. Comparative assessment of microscopy and rapid diagnostic test (RDT) as malaria diagnostic tools. Res J Parasitol. 2015;10:120-6.

41. Kim J-Y, Ji S-Y, Goo Y-K, Na B-K, Pyo H-J, Lee H-N, et al. Comparison of rapid diagnostic tests for the detection of Plasmodium vivax malaria in South Korea. PLoS One. 2013;8:e64353.

42. Djallé D, Gody JC, Moyen JM, Tekpa G, Ipero J, Madji N, et al. Performance of Parachecck ${ }^{\mathrm{TM}}$-Pf, SD Bioline malaria Ag-Pf and SD Bioline malaria Ag-Pf/ pan for diagnosis of falciparum malaria in the Central African Republic. BMC Infect Dis. 2014;14:109.

43. WHO. Malaria rapid diagnostic test performance: results of WHO product testing of malaria RDTs: round 1-7 (2008-2016). Geneva: World Health Organization; 2017.

44. Endeshaw T, Gebre T, Ngondi J, Graves PM, Shargie EB, Ejigsemahu Y, et al. Evaluation of light microscopy and rapid diagnostic test for the detection of malaria under operational field conditions: a household survey in Ethiopia. Malar J. 2008;7:118

45. Bisoffi Z, Sirima SB, Menten J, Pattaro C, Angheben A, Gobbi F, et al. Accuracy of a rapid diagnostic test on the diagnosis of malaria infection and of malaria-attributable fever during low and high transmission season in Burkina Faso. Malar J. 2010;9:192.
46. Wu L, Van Den Hoogen LL, Slater H, Walker PGT, Ghani AC, Drakeley CJ, et al. Comparison of diagnostics for the detection of asymptomatic Plasmodium falciparum infections to inform control and elimination strategies. Nature. 2015;528:86-93.

47. Mukadi P, Gillet P, Lukuka A, Atua B, Sheshe N, Kanza A, et al. External quality assessment of Giemsa-stained blood film microscopy for the diagnosis of malaria and sleeping sickness in the Democratic Republic of the Congo. Bull World Health Organ. 2013;91:441-8.

48. Mueller I, Betuela I, Ginny M, Reeder JC, Genton B. The sensitivity of the OptiMAL rapid diagnostic test to the presence of Plasmodium falciparum gametocytes compromises its ability to monitor treatment outcomes in an area of Papua New Guinea in which malaria is endemic. J Clin Microbiol. 2007:45:627-30.

49. Kozycki CT, Umulisa N, Rulisa S, Mwikarago El, Musabyimana JP, Habimana JP, et al. False-negative malaria rapid diagnostic tests in Rwanda: impact of Plasmodium falciparum isolates lacking hrp2 and declining malaria transmission. Malar J. 2017;16:123.

50. Gamboa D, Ho MF, Bendezu J, Torres K, Chiodini PL, Barnwell JW, et al. A large proportion of $P$. falciparum isolates in the Amazon region of Peru lack pfhrp2 and pfhrp3: implications for malaria rapid diagnostic tests. PLoS One. 2010;5:e8091.

51. Berhane A, Russom M, Bahta I, Hagos F, Ghirmai M, Uqubay S. Rapid diagnostic tests failing to detect Plasmodium falciparum infections in Eritrea: an investigation of reported false negative RDT results. Malar. J. 2017;16:105.

52. Soti DO, Kinoti SN, Omar AH, Logedi J, Mwendwa TK, Hirji Z, et al. Feasibility of an innovative electronic mobile system to assist health workers to collect accurate, complete and timely data in a malaria control programme in a remote setting in Kenya. Malar J. 2015;14:430.
Ready to submit your research? Choose BMC and benefit from:

- fast, convenient online submission

- thorough peer review by experienced researchers in your field

- rapid publication on acceptance

- support for research data, including large and complex data types

- gold Open Access which fosters wider collaboration and increased citations

- maximum visibility for your research: over $100 \mathrm{M}$ website views per year

At BMC, research is always in progress.

Learn more biomedcentral.com/submissions 